\title{
Progress in Chemokine-Like Factor 1 Research over the Past 10 Years: From Promoting Inflammatory Cell Trafficking to Inhibiting Allergic Airway Inflammation
}

\section{Ya-xia Tan}

The State Key Laboratory of Respiratory Disease, First Affiliated Hospital, Guangzhou Medical College, Guangzhou, China

\begin{abstract}
Chemokine-like factor 1 (CKLF1) is a cytokine, first described in 2001. CKLF1 is highly expressed in the lung and leukocytes. Recombinant CKLF1 has chemotactic activity on leukocytes, and stimulates the proliferation of murine skeletal muscle cells. Administration of CKLF1 in mice caused dramatic pathological changes in the lungs, including peribronchial leukocyte infiltration, epithelial shedding, collagen deposition, and proliferation of bronchial smooth muscle cells. Expression of CKLF1 mRNA in PBMCs and CKLF1 immunoreactivity in bronchial mucosa were found to be higher in an asthmatic group than in controls. A CKLF1-specific monoclonal antibody was generated using an intramuscular injection of pCDI-CKLF1 followed by electroporation in vivo, instead of a conventional protein immunization strategy. CCR4 is a functional receptor for CKLF1; this was confirmed using a chemotaxis assay, calcium flux assays, and receptor internalization. Two peptides from secreted CKLF1 in cell supernatants were obtained when recombinant CKLF1 was stably expressed in Drosophila S2 cells, termed CKLF1-C27 (C27) and CKLF1-C19 (C19). C27 and C19 had the same effect through CCR4 as the recombinant CKLF1 protein. Although with weaker chemotactic activity, C19 can inhibit chemotaxis induced by other chemokines, such as CKLF1 and TARC/CCL17. Chemically synthesized C19 peptide was injected intraperitoneally to inhibit allergic inflammation associated with asthma in mice, resulting in a significant reduction in AHR, airway eosinophilia, and the number of lymphocytes in BALF. Most recently, C19 peptide was used to treat murine allergic rhinitis. Intranasal administration of $\mathrm{C} 19$ reduced allergic symptoms, such as sneezing and rubbing, and serum concentrations of IgE. Mice treated with C19 or budesonide, a intranasal glucocorticoid steroid for the treatment of non-infectious rhinitis, for the treatment of non-infectious rhinitis, showed fewer eosinophils in the submucosa or peribronchiolar zone, while the nasal mucosa of untreated allergic mice displayed significant increases in eosinophils and conspicuous hyperplasia of the mucous glands.
\end{abstract}

Keywords: Chemokine; CKLF1; Electroporation; Airway inflammation; Airway remodeling; Allergic asthma; Peptide C19

\section{Introduction}

Cytokines are soluble proteins produced by nucleated cells throughout the body with essential roles in hemopoiesis, cell growth and differentiation, mediating inflammatory or immune responses, and tissue repair. In general, cytokines can be classified into the following groups: interleukins (IL), tumor necrosis factors (TNF), colony-stimulating factors (CSF), interferons (IFN), growth factors (GF) and chemokines [1-3]. The chemokines, as their name suggests, are chemotactic cytokines that direct the recruitment of immune cells and coordinate immune responses [3]. Chemokines can be classified into four families on the basis of the position of the first two cysteine residues: CXC-chemokine, CC-chemokine, $\mathrm{C}$-chemokine and $\mathrm{CX} 3 \mathrm{C}$ chemokine [4]. Because the chemokines are small proteins, the probability that their entire coding regions might be represented as expressed sequence tags (ESTs) in databases was very high [4]. Thus, we can use recombinant DNA technology and computer-assisted sequence analysis to rapidly identify and clone novel chemokine genes.

In this review, we introduce how skillfully applied advanced bioinformatics and genetic engineering technology were used to discover a novel cytokine, chemokine-like factor 1 (CKLF1) [5], and its MARVEL super family members [6]. We also demonstrated murine airway inflammation induced by CKLF1 [7], and that over-expression of CKLF1 was observed in asthmatics [8]. We also show how a monoclonal antibody to CKLF1 was generated [9], how its functional receptor was confirmed, and how two peptides were isolated from secreted CKLF1 $[10,11]$. Finally, we show that murine allergic inflammation in asthma and rhinitis can be inhibited by the $\mathrm{C} 19$ peptide, which could be a candidate for antagonizing allergic inflammation [12,13].

\section{Discovery of CKLF1 and Nomenclature}

In the 1990s, a new strategy for cloning novel cytokines was to retrieve known genes from gene sequence databases to discover homologous new genes, and then to clone and identify them. However, given that the human genetic database was publicly available on the Internet, this kind of strategy was often readily used in searching for new genes, leading to the possibility of the same gene being cloned by several laboratories almost simultaneously. Thus, Han, Ma, and colleagues designed a unique technical route to find novel cytokines using suppression subtractive hybridization ( $\mathrm{SSH}$ ) technology for screening and isolating new genes encoding cytokines inhibited by IL$10[5]$.

Previous research had demonstrated that U937 mononuclear cells with phytohemagglutinin (PHA) stimulation could produce many

*Corresponding author: Tan Ya-xia, The State Key Laboratory of Respiratory Disease, First Affiliated Hospital, Guangzhou Medical College, Guangzhou, China, Tel: +86 13650892623; E-mail: yaxiatan@yahoo.com.cn

Received December 24, 2011; Accepted April 25, 2012; Published April 29, 2012

Citation: Tan YX, (2012) Progress in Chemokine-Like Factor 1 Research over the Past 10 Years: From Promoting Inflammatory Cell Trafficking to Inhibiting Allergic Airway Inflammation. J Aller Ther S1:006. doi:10.4172/2155-6121.S1-006

Copyright: (c) 2012 Tan YX, et al. This is an open-access article distributed unde the terms of the Creative Commons Attribution License, which permits unrestricted use, distribution, and reproduction in any medium, provided the original author and source are credited. 
kinds of cytokines, while interleukin 10 (IL-10) was known to inhibit the generation of various cytokines $[14,15]$. Based on these two facts, Han suggested that the cytokines in PHA-stimulated U937 cells could be inhibited by IL-10 and among the inhibited cytokines, there was a likelihood that some of the genes would not have been characterized previously [5]. Thus, they used PHA-stimulated U937 cells as the tester and PHA-stimulated and simultaneously IL-10-inhibited U937 cells as the driver in the SSH procedure [5]. Eventually a novel gene that was inhibited by IL-10 was isolated. The full-length cDNA contained 530 base pairs and encoded a single open reading frame of 99 amino acid residues, including a CC motif similar to those of other CC family chemokines. Therefore, the gene represented a novel CC family chemokine, and its GenBank accession number was AF096985 [5].

Because the novel gene was cloned from U937 cells and contained a CC motif with the chemotactic effects on leucocytes, it was then named U937-derived chemokine, abbreviated as UCK. However, UCK differed from classical CC chemokines for the following reasons: 1) there was no additional two C-terminus cysteines in the mature protein, although there was one conserved CC motif, 2) there were three other RNA splicing isoforms of $U C K$, encoding 152, 67, and 120 amino acid variants, and they shared the same amino- and carboxylterminal amino acid sequences as UCK, and 3) it could stimulate the proliferation and differentiation of skeletal muscle cells.

Therefore, following a recommendation from Dr. Julia White of University College, London, UK, and the Human Genome Organization Nomenclature Committee (HGONC), the name UCK was changed to "chemokine-like factor 1" (CKLF1). Thus, CKLF1 possesses certain special structures and potentially more extensive functions beyond its effects in chemotaxis. Also, its three isoforms were named CKLF2, CKLF3, and CKLF4. Further experimental data showed that CKLF1 was a secreted protein, whereas CKLF2 and CKLF4 were transmembrane proteins [5].

CKLF1 is highly expressed in multiple tissues and cells, such as lung and leukocytes. Recombinant CKLF1 exhibits potent chemotactic activity on leukocytes and lymphocytes in vitro and in vivo. Moreover, it stimulates the proliferation of murine skeletal muscle cells after in vivo administration of CKLF1 plasmid DNA [5].

\section{Identification of Chemokine-Like Factor Superfamily Members: CMTM 18}

Before CKLF was discovered in 1998, no one had described a human gene product with obvious sequence similarity to CKLF. According to Zlotnik, there would be expected to be a high frequency of gene duplication within structurally similar chemokine clusters [4]. Thus, it was reasonable to believe that additional genes related to CKLF1 could exist.

The gene encoding CKLF is located on chromosome $16 \mathrm{q} 22$. Thus, when it came to chemokines on chromosome 16q, three other well-known chemokines and one transmembrane protein were vital clues. The genes encoding chemokine CCL22/MDC, CCL17/TARC, and CX3CL1/fractalkine, and the transmembrane 4 superfamily 11 protein (TM4SF11) are clustered on chromosome 16q13 [16,17]. Although TM4SF11 is not a chemokine, it is a shorter gene product from CCL22/MDC, and its encoding gene is also at chromosome $16 q 13$ [17]. Moreover, the key amino acids of CCL22/MDC and CCL17, which are around a gene-specific motif, are identical to those of CKLF. Most importantly, CKLF has four alternative RNA splicing forms, CKLF1, 2, 3, and 4. Among these four isoforms, CKLF2 is the full-length cDNA product, encoding 152 amino acids and has four putative transmembrane regions $[5,18]$. The sequence identity between CKLF2 and TM4SF11 is $15.4 \%$. All of this information indicated that the CKLF gene may be an interim gene, between the CCL22/MDC, CCL17/TARC, and TM4SF11 genes.

Next, using a computational search strategy combined with genetic engineering technology and bioinformatics analysis [19], Hang and Ding cloned and identified eight further novel genes related to CKLF that formed gene clusters, and were designated "chemokine-like factor superfamily members 1-8" (CKLFSF1-8) [6]. In 2005, according to the structural characteristics of the members of the family, the HGONC decided to rename CKLFSF1-8 as "CKLF-like MARVEL transmembrane domain-containing 1-8" (CMTM1-8), indicating that CMTM represented a novel gene family between the SCY and the TM4SF gene families. In humans, the family includes nine genes, CKLF and CMTM 1-8. However, in this paper we will focus on research about CKLF1.

\section{Effects of CKLF1 on Airway Inflammation and Remodeling}

Because CKLF1 is expressed in the lung and recombinant CKLF1 exhibited chemotactic effects on human leukocyte recruitment, and stimulated the proliferation of skeletal muscle, we hypothesized that airway structural and inflammatory cells may use CKLF1 to regulate leukocyte trafficking and induce tissue cell proliferation, which could be involved in the pathogenesis of important pulmonary diseases, such as asthma. Asthma is characterized by the persistent aggregation of inflammatory cells, which is associated with epithelial cell damage, collagen deposition, and proliferation of fibroblasts and smooth muscle cells [20].

Thus, we constructed a CKLF1-expressing plasmid, pCDI-CKLF1, and evaluated the biological effect of CKLF1 in murinelung using a single intramuscular injection of pCDI-CKLF1, followed by electroporation, a highly efficient method for delivering genes into skeletal myofibers to produce high levels of protein in circulating plasma [21-23]. At 1 week after intramuscular CKLF1 plasmid injection, strong expression of the exogenous CKLF1 protein in murine lung tissue was visualized by Western blotting, indicating that the CKLF1 protein was effectively delivered to the lungs as a result of electroporated plasmid injection [7], and the subsequent dramatic pathological changes in the lungs, including peribronchial leukocyte infiltration, collagen deposition, and proliferation of bronchial smooth muscle cells, were caused by over expression of CKLF1 [7].

The morphological changes in inflammation and proliferation in the lung induced by CKLF1 were similar to phenomena observed in chronic persistent asthma. Furthermore, expression of CKLF1 mRNA was measured in PBMCs and CKLF1 protein in bronchial tissues was assessed in patients with asthma. The results showed that expression of CKLF1 mRNA in PBMCs and CKLF1 immunoreactivity in bronchial mucosa was both higher in the asthmatic group than in the controls [8].

Overall, the inflammatory process and subsequent subepithelial changes initiated by CKLF1 seemed to mimic the airway remodeling seen in chronic bronchial asthma, and over-expression of CKLF1 was indeed observed in asthmatics. These findings supported Holgate's 
theory of the "reactivation of the Epithelial-Mesenchymal Trophic Unit (EMTU)" in the pathogenesis of asthma. This theory emphasized that chronic asthma is characterized by enhanced epithelial-mesenchymal communication with the release of a range of different growth factors linked to airway remodeling $[24,25]$.

\section{Quality of the Antibody Generated against Recombinant CKLF1}

To further study the structural and functional characteristics of CKLF1, it was necessary to make an antibody against CKLF1. A recombinant CKLF1 plasmid was constructed and expressed in E. coli with high efficiency in the lab of the Center for Human Disease Genomics, Peking University, Beijing. After purification, recombinant CKLF protein was obtained, and, subsequently, following conventional hybridoma techniques, a monoclonal antibody against recombinant CKLF1 was prepared. Using the specific antibody, we detected the over-expression of CKLF1 protein in bronchial tissues in asthmatics [8].

However, CKLF1 is a highly hydrophobic protein and the purification of native CKLF1 was unsuccessful. Receiving inspiration from the advantages of DNA immunization for antibody production and our previous discovery injecting the pCDI-CKLF1 plasmid into the skeletal muscle of mice with electroporation, leading to secretion of the protein in vivo and subsequently causing dramatic pathological changes in the murine lung [7,26-28], Chen used the method of injecting plasmid DNA in vivo to generate an antibody in vivo instead of a conventional protein immunization strategy [9].

Briefly, a dose of DNA $(100 \mu \mathrm{g})$ in $0.9 \% \mathrm{NaCl}(100 \mu \mathrm{L})$ was injected into an anesthetized animal followed by electric pulses. Mice were immunized three times at 3-week intervals. Hybridomas were generated by the fusion of myeloma SP2/O cells with mouse spleen cells. One hybridoma cell line stably secreting a monoclonal antibody against CKLF1 was generated and designated "clone M4." The specificity was assessed by enzyme-linked immunosorbent assay (ELISA) and Western blotting.

The M4 monoclonal antibody was also found to be immunoglobulin subclass IgG1 and specific for the unique C-terminal domain of CKLF proteins [9]. These results demonstrated that intramuscular injection of naked DNA encoding a target gene, combined with electroporation in vivo, can be used to prepare monoclonal antibodies.

Trollet et al. [29] and Michele et al. [30] made the following comments regarding this effective and simple method: "A very interesting use of electrotransfer described the preparation of a monoclonal antibody against CKLF1 (chemokine like factor 1), a newly cloned human cytokine. As CKLF1 is a highly hydrophobic protein, the purification of native CKLF1 was unsuccessful. Electrotransfer of a CKLF1 encoding plasmid into the skeletal muscle of mice (and therefore in vivo secretion of the protein) instead of the conventional protein immunization strategy overcame this problem led to the generation of the desired monoclonal antibody" [29], and "In vivo expression levels improved markedly using this approach - levels increased by several fold over plasmid injection alone. This method might allow for less frequent immunizations with the DNA platform, and can improved both cellular and humoral responses" [30]. Since then, the monoclonal antibody against CKLF1 has been used in immunohistochemistry, Western blotting, and to neutralize the chemotactic effect of CKLF1.

\section{Confirmation of CCR4 as a Functional Receptor for CKLF1}

It is only when a chemokine binds to its specific receptor on a target cell that it can have biological activity [4]. All chemokines mediate their activities via uniting a corresponding heterotrimeric G-protein-coupled receptor, generating signals subsequently transduced to the inside of the cell $[31,32]$. Although previous research had demonstrated that CKLF1 exerted multiple activities, it was not known which receptor(s) possessed specific affinity for CKLF1. Consistent with the state of background knowledge, Wang and Zhang performed interesting and highly effective work to evaluate the receptor [10]. The work was based on the following clues. 1) About 50 chemokines and 20 receptors have been identified so far; that is, more chemokines and fewer receptors, suggesting that a number of chemokines might share the same receptor [4]. Thus it was possible that CKLF1 shared a receptor with other related chemokines. 2) CKLF1 possessed similarities in structure, chromosomal, location and functional properties with CC chemokines TARC/CCL17 and MDC/CCL22, which bind to CC chemokine receptor type 4 (CCR4) to exert their function $[33,34]$. Thus, it seemed possible that, like TARC/CCL17 and MDC/CCL22, CKLF1 might interact with CCR4. 3) CKLF1 causes dramatic airway inflammation and remodeling in the murine lung, mimicking the pathology seen in chronic persistent asthma, and over-expression of CKLF1 was detected in asthmatics [7,8]. 4) Expression of CKLF1 was up-regulated during T lymphocyte activation [35], and CCR4 is highly expressed in Th2 lymphocytes and the interaction of CCR4 with its ligands induced the migration of Th2 cells, playing an important role in the pathogenesis of asthma [36,37]. Therefore, Wang and Zhang subsequently targeted CCR4 and carried out the following systematic experiments, finally proving the hypothesis [10].

Cloned CCR4 from a K562 cell cDNA library was expressed efficiently in human embryonic kidney 293 (HEK293) cells, which are easy to grow and transfect. Recombinant CKLF1 was secreted using a Drosophila expression system [10]. CCR6- expressing and mock-transfected cells were also cloned as controls. The interaction of CKLF1 with CCR4 was confirmed by means of a chemotaxis assay, an intracellular calcium flux assay, and receptor internalization. In the chemotaxis assay, when treated with CKLF1, pcDI-CCR4-transfected HEK293 cells produced classic bell-shaped migration responses while pcDI-CCR6-transfected cells failed to migrate [10]. Pretreatment with TARC/CCL17 can desensitize CCR4 to subsequent stimulation with CKLF1. In calcium mobilization, with the same effect as TARC/CCL17, CKLF1 also induced calcium flux specifically in CCR4-transfected HEK293 cells, but not in control cells [10]. Moreover, CKLF1 completely desensitized CCR4 transfectants to subsequent TARC/ CCL17 treatment [10].

According to a report that after HEK293 cells were transfected with the pEGFP-N1 vector, EGFP protein was expressed in the cytosol of the cells [38], Wang finally conducted a receptor internalization assay with HEK293 cells transiently expressing pCCR4-EGFP or pCCR6EGFP, and the subcellular localization of the CCR4-EGFP or CCR6EGFP protein was visualized using confocal fluorescence microscopy. The data showed that the CCR4-EGFP fusion protein was expressed in the cytosol of the cells treated with CKLF1, but CCR6-EGFP was not, indicating that CKLF1 induced the internalization of CCR4 [10]. On the basis that CKLF1 induced chemotaxis, calcium flux, and internalization specifically in CCR4-transfected cells, it was thus confirmed that CCR4 was a functional receptor for CKLF1. 
Citation: Tan YX, (2012) Progress in Chemokine-Like Factor 1 Research over the Past 10 Years: From Promoting Inflammatory Cell Trafficking to Inhibiting Allergic Airway Inflammation. J Aller Ther S1:006. doi:10.4172/2155-6121.S1-006

\section{Isolation of Two Peptides from Secreted CKLF1}

CKLF1 is a highly hydrophobic protein and the purification of native CKLF1 was unsuccessful. However, when recombinant CKLF1 was stably expressed in Drosophila S2 cells, two peptides from secreted CKLF1 in the transfected cell supernatant were obtained and were stained with Coomassie Brilliant Blue R-250; they were named CKLF1-C27 (C27) and CKLF1-C19 (C19). N-terminal amino acid sequencing showed that the peptide sequence of $\mathrm{C} 27$ was ALIYRKLLFNPSGPYQKKPVHEKKEVL and that of C19 was FNPSGPYQKKPVHEKKEVL [11].

Because CKLF1 exerts its effects via CCR4 [10], it was reasonable to consider whether C27 and C19 from secreted CKLF1 also caused the same effects as the recombinant CKLF1 protein through CCR4. Thus, Wang and Zhang next tested the biological functions of chemically synthesized peptides C27 and C19 in terms of chemotaxis, calcium mobilization, and receptor internalization assays in CCR4-transfected HEK293 or the human T cell line Hut78 cells, with strong endogenous expression of CCR4 induced by the TARC/CCL17 protein. Wang and Zhang showed that both C27 and C19 induced chemotaxis and calcium flux in CCR4-transfected HEK293 cells, but not in mock-transfected cells as a control [11]. After HEK293 cells were transfected with the CCR4-EGFP plasmid, a CCR4-EGFP fusion protein was expressed at the plasma membrane, and when treated with C27 and C19, the EGFP protein was seen in the cytosol of the cells and clear internalization of CCR4-EGFP was visualized using confocal fluorescence microscopy [11]. Furthermore, the chemotactic response induced by C19 and C27 was markedly inhibited by preincubation with a CCR4 antagonist. Thus, these results demonstrate that like CKLF1, C27 and C19 could induce chemotaxis, calcium mobilization, and receptor internalization, and interact with CCR4 [11].

A surprising discovery during these experiments was the difference in the degree of chemotaxis between C27 and C19. C27 significantly induced chemotaxis in CCR4-transfected Hut78 cells, while C19 showed weak chemotactic activity in these cells [11]. This differing degree of chemotaxis between C19 and C27 in endogenously CCR4expressing Hut78 cells raised the question as to whether the two peptides might have differing physiological, or pathophysiological, functions in vivo.

\section{Inhibition of Allergic Asthma and Rhinitis with CKLF1-C19 Peptide in Mice}

As mentioned above, like CKLF1, the CKLF1 C-terminal peptides C27 and C19 also interact with human CCR4, but C19 weakly activates CCR4 signaling and may even be a candidate antagonist peptide of CCR4, because C19 desensitized the chemotaxis induced by TARC/ CCL17 via CCR4, the common receptor shared by C19, CKLF1, and TARC/CCL17, indicating that C19 can inhibit chemotaxis induced by other chemokines, such as CKLF1 and TARC/CCL17 [11]. Thus, Tian, Li and Wang hypothesized that $\mathrm{C} 19$ might act as an antagonist in the development of asthma in mice [12]. Murine allergic asthma was generated by sensitizing with intraperitoneal injections of ovalbumin (OVA) and challenging the mice with inhaled aerosolized OVA. At 30 min before each OVA challenge, chemically synthesized C19 peptide, dissolved in saline, was injected intraperitoneally.

The inhibitory effects of $\mathrm{C} 19$ in the pathogenesis of allergic asthma focused on AHR, eosinophil infiltration, and pathological changes in the lung. The data showed that, compared with the OVA group treated with saline, treatment with $\mathrm{C} 19$ resulted in a significant reduction in
AHR, airway eosinophilia, and inflammation [12]. Additionally, C19 inhibited chemotaxis mediated via CCR4 in human Th2 lymphocytes and in splenocytes from ovalbumin-sensitized mice [12].

In addition to CCR4, CC chemokine receptor 3 (CCR3), the principal chemokine receptor expressed by eosinophils, is involved in airway eosinophilia and hyper-responsiveness in allergic asthma $[39,40]$. CCR3 mediates the biological effects of important CCchemokines, such as CCL5, -7,-11,-13, -24, and -26 [41,42]. Therefore, Tian, Li and Wang constructed expression plasmids for human CCR3 and mouse Ccr3, and then transfected them into HEK293 cells. Prior to stimulation with human CCL11, the cells were pretreated with $\mathrm{C} 19$ for $30 \mathrm{~min}$. Then, peritoneal cells or splenocytes from OVA-sensitized mice were used to examine chemotaxis [12].

The data showed that $\mathrm{C} 19$ inhibited the chemotaxis mediated by CCR3 in peritoneal cells or splenocytes. Moreover, C19 inhibited CCL11-induced chemotaxis of mouse eosinophils and human CCR3transfected or mouse Ccr3-transfected HEK293 cells.

That is, C19 attenuated airway eosinophilia and lung inflammation by inhibiting CCR4- and CCR3-mediated chemotaxis in a mouse model of asthma. Because of this ability to attenuate airway eosinophilia and lung inflammation, by inhibiting CCR4- and CCR3-mediated chemotaxis, $\mathrm{C} 19$ has great therapeutic potential for use in the treatment and control of allergic asthma.

Most recently, Zheng and colleagues reported that they had applied peptide C19 intranasally to treat allergic rhinitis in mice [13] Like allergic asthma, allergic rhinitis also involves activation of CCR3 and CCR4 $[43,44]$. A mouse model of allergic rhinitis was immunized intraperitoneally with OVA and challenged locally with OVA in their nostrils. At 30 min before intranasal OVA challenge, selected mice were treated with $\mathrm{C} 19$ or budesonide (intranasal steroid) intranasally. Intranasal administration of $\mathrm{C} 19$ reduced allergic symptoms, such as sneezing and rubbing, and the serum concentration of IgE [13]. The nasal mucosa of untreated allergic mice displayed a significant increase in eosinophils and conspicuous hyperplasia of the mucous glands, while mice treated with $\mathrm{C} 19$ or budesonide showed fewer eosinophils in the submucosa or peribronchiolar zone. Peptide C19 intranasal administration had a strong therapeutic effect and was found to be as effective against allergic rhinitis as budesonide, which may suggest potential in the treatment of allergic rhinitis.

\section{Determination of the Molecular Mechanism of the Suppression of Airway Inflammation by CKLF1-C19 Peptide}

The studies discussed above show that CKLF1-C19 peptide could inhibit inflammation in allergic asthma and rhinitis in mice, while the CKLF1-C27 peptide promoted inflammation (unpublished data). Why may CKLF1-C19 be a candidate inflammation inhibitor? Liu Yi et al. solved the mystery using capillary zone electrophoresis (CZE) to analyze interactions between CKLF1 peptides and heparin [45].

Most chemokines in vivo bind not only to their receptors but also to glycosaminoglycans (GAGs), such as heparin, to exert their biological activity on target cells [46-48]. The two CKLF1 peptides with positive charges due to basic amino acids can bind to the GAGs, with negative charges due to acidic amino acid. Chemokine immobilization through the GAG interaction facilitates the formation of haptotactic chemokine gradients, and enhances their concentration at the site of production. By binding to GAGs, which protects chemokines from 
Citation: Tan YX, (2012) Progress in Chemokine-Like Factor 1 Research over the Past 10 Years: From Promoting Inflammatory Cell Trafficking to Inhibiting Allergic Airway Inflammation. J Aller Ther S1:006. doi:10.4172/2155-6121.S1-006

degradation, chemokines maintain the lifetime and availability in vivo $[49,50]$. Because of low sample consumption, easy calculation of binding constants, and simple operating procedure, CZE is an effective strategy for analyzing interactions between chemokines and heparin, which is a kind of free GAG existing in plasma and is widely available commercially [51,52].

Evaluation of the interactions between heparin and C27/C19, based on CZE, demonstrated that C19 and C27 can interact with heparin, and the binding constant of $\mathrm{C} 27$ is higher than that of $\mathrm{C} 19$, which is in favor of the combination of $\mathrm{C} 27$ and CCR4, leading to strong chemotactic activity, whereas the combination of C19 and CCR4 is less favored, because $\mathrm{C} 19$ has a weaker ability to bind heparin. Compared with C27, C19 lacks eight amino acids at its N-terminus and has weaker ability in heparin binding, which may be one reason for CKLF1-C19 antagonizing inflammation [45].

\section{Epilogue}

In this review, I have told the interesting story of the progress in CKLF1 research over the past 10 years. In 2001, the first report about the discovery of a novel cytokine, CKLF1, was published. In December 2011, the CKLF1-C19 peptide's effects against allergic inflammation were reported. During the 10 years, researchers in the fields of immunology, respiratory disease, and pharmacy collaborated with one another and successfully carried out a series of experiments, from the cloning of CKLF1 to intervention against allergic airway inflammation.

In Chinese laboratories, CKLF1 was the first chemotactic cytokine to be cloned by Chinese immunologists. Immediately after CKLF1 was discovered, specialists in respiratory diseases introduced CKLF1 in research in asthmatics because of the effects of CKLF1 on inflammatory cell trafficking and tissue cell proliferation. They demonstrated that CKLF1 was involved in asthmatics and caused airway inflammation and remodeling. The immunologists went on to finish the identification of CKLF1 superfamily members and generated a monoclonal antibody against CKLF1. Then, they focused on a link between CKLF1 and asthma to confirm CCR4 as a functional receptor for CKLF1 and isolated two peptides from secreted CKLF1, C27 and C19, and suggested that C19 had anti-inflammatory effects, finally revealing that CKLF1-C19 peptide, a small molecule inhibitor, may have efficacy in the treatment of allergic asthma and rhinitis.

The past 10 years have been a very exciting time in CKLF1 research. We have witnessed the discovery and characterization of CKLF1, from promoting inflammatory cells trafficking to inhibiting allergic airway inflammation. Looking at the development of CKLF1, it provides an important example for searching for other novel genes that remain to be discovered and characterized. As in the case of CKLF1, exploring novel genes needs extensive cooperation in a number of areas using new technologies, such as genomics and bioinformatics, and new knowledge about related diseases and pharmacology.

\section{Acknowledgements}

This work was supported by the National Natural Science Foundation of China (No. 30370622), the Guangdong Natural Science Foundation Key Program (No.020741) and the State Key Laboratory of Respiratory Disease Foundation (2010).

\section{References}

1. Corwin EJ (2000) Understanding Cytokines. Part I: Physiology and Mechanism of Action. Biol Res Nurs 2: 30-40.

2. Oppenheim JJ (2001) Cytokines: past, present, and future. Int J Hematol 74: 3-8

3. Cascieri MA, Springer MS (2000) The chemokine/chemokine-receptor family: potential and progress for therapeutic intervention. Curr Opin Chem Biol 4 420-427.

4. Zlotnik A, Yoshie O (2000) Chemokines: A new classification system and their role in immunity. Immunity 12: 121-127.

5. Han W, Lou Y, Tang J, Zhang Y, Chen Y, et al. (2001) Molecular cloning and characterization of chemokine-like factor 1 (CKLF1), a novel human cytokine with unique structure and potential chemotactic activity. Biochem J 357: 127 135

6. Han W, Ding P, Xu M, Wang L, Rui M, et al. (2003) Identification of eight genes encoding chemokine-like factor superfamily members 1-8 (CKLFSF1-8) by in silico cloning and experimental validation. Genomics 81: 609-617.

7. Tan YX, Han WL, Chen YY, Ouyang NT, Tang Y, et al. (2004) Chemokinelike factor 1 , a novel cytokine, contributes to airway damage, remodeling and pulmonary fibrosis. Chin Med J 117: 1123-1129

8. Tan YX, Ma DL, Han W, Chen Y, Zhong N (2001) Chemokine-Like Factor 1, A Novel Human Cytokine, Contributes To The Airway Remodeling In Asthma. Abstract Book, 3rd Triennial World Asthma Meeting, Chicago, USA.

9. Chen Y, Zhang T, Li T, Han W, Zhang Y, et al. (2005) Preparation and characterization of monoclonal antibody against CKLF using DNA immunization with in vivo electroporation. Hybridoma (Larchmt) 24: 305-308.

10. Wang Y, Zhang Y, Yang X, Han W, Liu Y, et al. (2006) Chemokine-Like Factor 1 Is a Functional Ligand for CC Chemokine Receptor 4 (CCR4). Life Sci 78 : 614-621.

11. Wang Y, Zhang Y, Han W, Li D, Tian L, et al. (2008) Two C-terminal peptides of human CKLF1 interact with chemokine receptor CCR4. Int J Biochem Cell Biol 40: 909-919.

12. Tian L, Li W, Wang J, Zhang Y, Zheng Y, et al. (2011) The CKLF1-C19 peptide attenuates allergic lung inflammation by inhibiting CCR3- and CCR4-mediated chemotaxis in a mouse model of asthma. Allergy 66: 287-297.

13. Zheng $Y$, Guo C, Zhang $Y$, Qi H, Sun Q, et al. (2011) Alleviation of murine allergic rhinitis by $\mathrm{C} 19$, a C-terminal peptide of chemokine-like factor 1 (CKLF1). Int Immunopharmacol 11: 2188-2193.

14. Brantschen S, Gauchat JF, de Weck AL, Stadler BM (1989) Differentia expression of cytokine mRNAs in human cell lines. Lymphokine Res 8: 163 172

15. de Waal Malefyt R, Abrams J, Bennett B, Figdor CG, de Vries JE (1991) Interleukin-10 (IL-10) inhibits cytokine synthesis by human monocytes: an autoregulatory role of IL-10 produced by monocytes. J Exp Med 174: 12091220.

16. Nomiyama H, Imai T, Kusuda J, Miura R, Callen DF, et al. (1998) Human chemokines fractalkine (SCYD1), MDC (SCYA22) and TARC (SCYA17) are clustered on chromosome 16q13. Cytogenet Cell Genet 81: 10-11.

17. Hamacher M, Pippirs U, Köhler A, Müller HW, Bosse F (2001) Plasmolipin: genomic structure, chromosomal localization, protein expression pattern, and putative association with Bardet-Biedl syndrome. Mamm Genome 12: 933-937.

18. Xia D, Li X, Lou Y, Han W, Ding P, et al. (2002) Overexpression of chemokinelike factor 2 promotes the proliferation and survival of $\mathrm{C} 2 \mathrm{C} 12$ skeletal muscle cells. Biochim Biophys Acta 1591: 163-173.

19. Schutte BC, Mitros JP, Bartlett JA, Walters JD, Jia HP, et al. (2002) Discovery of five conserved defensin gene clusters using a computational search strategy. Proc Natl Acad Sci U S A 99: 2129-2133.

20. Bousquet J, Jeffery PK, Busse WW, Johnson M, Vignola AM (2000) Asthma. From bronchoconstriction to airways inflammation and remodeling. Am J Respir Crit Care Med 161: 1720-1745

21. Wolff JA, Malone RW, Williams P, Chong W, Acsadi G, et al. (1990) Direct gene transfer into mouse muscle in vivo. Science 247: 1465-1468.

22. Aihara H, Miyazaki J (1998) Gene transfer into muscle by electroporation in vivo. Nat Biotechnol 16: 867-870.

23. Bettan M, Emmanuel F, Darteil R, Caillaud JM, Soubrier F, et al. (2000) Highlevel protein secretion into blood circulation after electric pulse-mediated gene transfer into skeletal muscle. Mol Ther 2: 204-210.

24. Holgate ST, Davies DE, Lackie PM, Wilson SJ, Puddicombe SM, et al. (2000) Epithelial-mesenchymal interactions in the pathogenesis of asthma. J Allergy Clin Immunol 105: 193-204. 
Citation: Tan YX, (2012) Progress in Chemokine-Like Factor 1 Research over the Past 10 Years: From Promoting Inflammatory Cell Trafficking to Inhibiting Allergic Airway Inflammation. J Aller Ther S1:006. doi:10.4172/2155-6121.S1-006

25. Holgate ST, Arshad HS, Roberts GC, Howarth PH, Thurner P, et al. (2009) A new look at the pathogenesis of asthma. Clin Sci (Lond) 118: 439-450.

26. Tang DC, DeVit M, Johnston SA (1992) Genetic immunization is a simple method for eliciting an immune response. Nature 356: 152-154.

27. Krasemann S, Jurgens T, Bodemer W (1999) Generation of monoclonal antibodies against prion proteins with an unconventional nucleic acid-based immunization strategy. J Biotechnol 73: 119-129.

28. Mir LM, Bureau MF, Gehl J, Rangara R, Rouy D, et al. (1999) High-efficiency gene transfer into skeletal muscle mediated by electric pulses. Proc Natl Acad Sci U S A 96: 4262-4267.

29. Trollet C, Bloquel C, Scherman D, Bigey P (2006) Electrotransfer into Skeletal Muscle for Protein Expression. Curr Gene Ther 6: 561-578.

30.Kutzler MA, Weiner DB (2008) DNA vaccines: ready for prime time? Nat Rev Genet 9: 776-788.

31. Oh SB, Endoh T, Simen AA, Ren D, Miller RJ (2002) Regulation of calcium currents by chemokines and their receptors. J Neuroimmunol 123: 66-75.

32. New DC, Wong YH (2003) CC chemokine receptor-coupled signalling pathways. Sheng Wu Hua Xue Yu Sheng Wu Wu Li Xue Bao (Shanghai) 35 779-788.

33. Imai T, Chantry D, Raport CJ, Wood CL, Nishimura M, et al. (1998) Macrophagederived chemokine is a functional ligand for the CC chemokine receptor 4 . J Biol Chem 273: 1764-1768.

34. Imai T, Nagira M, Takagi S, Kakizaki M, Nishimura M, et al. (1999) Selective recruitment of CCR4-bearing Th2 cells toward antigen-presenting cells by the CC chemokines thymus and activation-regulated chemokine and macrophagederived chemokine. Int Immunol 11: 81-88.

35. Li T, Zhong J, Chen Y, Qiu X, Zhang T, et al. (2006) Expression of chemokinelike factor 1 is upregulated during $T$ lymphocyte activation. Life Sciences 79 : 519-524.

36. Dauqherty BL, Siciliano SJ, DeMartino JA, Malkowitz L, Sirotina A, et al. (1996) Cloning, expression, and characterization of the human eosinophil eotaxin receptor. J Exp Med 183: 2349-2354.

37. Ponath PD, Qin S, Post TW, Wang J, Wu L, et al. (1996) Molecular cloning and characterization of a human eotaxin receptor expressed selectively on eosinophils. J Exp Med 183: 2437-2448.

38.Xu Y, Zhu K, Hong G, Wu W, Baudhuin LM, et al. (2000) Sphingosylphosphorylcholine is a ligand for ovarian cancer G-protein-coupled receptor 1. Nat Cell Biol 2: 261-267.

39. Forssmann U, Uquccioni M, Loetscher P, Dahinden CA, Lanqen H, et al. (1997) Eotaxin-2, a novel CC chemokine that is selective for the chemokine receptor
CCR3, and acts like eotaxin on human eosinophil and basophil leukocytes. $J$ Exp Med 185: 2171-2176.

40. Bisset LR, Schmid-Grendelmeier P (2005) Chemokines and their receptors in the pathogenesis of allergic asthma: progress and perspective. Curr Opin Pulm Med 11: 35-42.

41. Shinkai A, Yoshisue H, Koike M, Shoji E, Nakagawa S, et al. (1999) A nove human CC chemokine, eotaxin-3, which is expressed in IL-4-stimulated vascular endothelial cells, exhibits potent activity toward eosinophils. J Immunol 163: $1602-1610$.

42. Shinkai A, Yoshisue H, Koike M, Shoji E, Nakagawa S, et al. (1999) A nove human CC chemokine, eotaxin-3, which is expressed in IL-4-stimulated vascular endothelial cells, exhibits potent activity toward eosinophils. J Immuno 163: $1602-1610$

43. Willems LI, ljzerman AP (2010) Small molecule antagonists for chemokine CCR3 receptors. Med Res Rev 30: 778-817.

44. Banfield G, Watanabe H, Scadding G, Jacobson MR, Till SJ, et al. (2010) CC chemokine receptor 4 (CCR4) in human allergen-induced late nasal responses. Allergy 65: 1126-1133.

45. Liu Y, Zhang S, Ling X, Li Y, Zhang Y, et al. (2008) Analysis of the interactions between the peptides from secreted human CKLF1 and heparin using capillary zone electrophoresis. J Pept Sci 14: 984-988.

46. Wagner L, Yang OO, Garcia-Zepeda EA, Ge Y, Kalams SA, et al. (1998) Beta-chemokines are released from HIV-1-specific cytolytic T-cell granules complexed to proteoglycans. Nature 391: 908-911.

47. Kuschert GS, Coulin F, Power CA, Proudfoot AE, Hubbard RE, et al. (1999) Glycosaminoglycans interact selectively with chemokines and modulate receptor binding and cellular responses. Biochemistry 38: 12959-12968.

48. Shriver Z, Liu D, Sasisekharan R (2002) Emerging views of heparin sulfate glycosaminoglycan structure/activity relationships modulating dynamic biological functions. Trends Cardiovasc Med 12: 71-77.

49. Sadir R, Imberty A, Baleux F, Lortat-Jacob H (2004) Heparin sulfate/heparin oligosaccharides protect stromal cell-derived factor-1 (SDF-1)/CXCL12 agains proteolysis induced by CD26/dipeptidyl peptidase IV. J Biol Chem 279: 4385443860.

50. Witt DP, Lander AD (1994) Differential binding of chemokines to glycosaminoglycan subpopulations. Curr Biol 4: 394-400

51. Heegaard $\mathrm{NH}$ (1998) Capillary electrophoresis for the study of affinity interactions. J Mol Recognit 11: 141-148.

52. Sun Z, Ling X, Sun W, Xiao J, Yin C, et al. (2007) Studies on the interaction between lactam analogs and the $\mathrm{N}$-terminal extracellular tail of CC chemokine receptor 4 by capillary zone electrophoresis. Electrophoresis 28: 3064-3069.
This article was originally published in a special issue, Airway Inflammation and Hyper responsiveness handled by Editor(s). Dr. John F. Alcorn (1), Children's Hospital of Pittsburgh, USA. 